\title{
Renin-angiotensin system blockers and mortality in COVID-19: a territory-wide study from Hong Kong
}

\author{
Jiandong Zhou MSc $\#^{1}$, Gary Tse PhD FRCP FFPH \# ${ }^{2}$, Sharen Lee ${ }^{3}$, Wing Tak Wong PhD ${ }^{4}$, \\ Xingsong Wang MSc ${ }^{5}$, William KK Wu PhD FRCPath FRCP ${ }^{5}$, Tong Liu MD PhD ${ }^{1}$, Zhidong Cao \\ $\mathrm{PhD}^{6}$, Daniel Dajun Zeng $\mathrm{PhD}^{6}$, Ian Chi Kei Wong $\mathrm{PhD}^{7}$, Bernard Man Yung Cheung MB BChir \\ PhD FRCP ${ }^{8}$, Qingpeng Zhang $\mathrm{PhD}^{1}$ \\ ${ }^{1}$ School of Data Science, City University of Hong Kong, Hong Kong, Hong Kong, China \\ 2 Tianjin Key Laboratory of Ionic-Molecular Function of Cardiovascular Disease, Department of \\ Cardiology, Tianjin Institute of Cardiology, Second Hospital of Tianjin Medical University, Tianjin \\ 300211, China \\ ${ }^{3}$ Laboratory of Cardiovascular Physiology, Li Ka Shing Institute of Health Sciences, China \\ ${ }^{4}$ School of Life Sciences, The Chinese University of Hong Kong, Hong Kong, China \\ ${ }^{5}$ Department of Anaesthesia and Intensive Care, Li Ka Shing Institute of Health Sciences, Hong \\ Kong, China \\ ${ }^{6}$ Institute of Automation, Chinese Academy of Sciences, Beijing, China. \\ ${ }^{7}$ Department of Pharmacology and Pharmacy, University of Hong Kong, Pokfulam, Hong Kong, \\ China \\ ${ }^{8}$ Division of Clinical Pharmacology and Therapeutics, Department of Medicine, The University of \\ \# joint first authors \\ Hong Kong, Pokfulam, Hong Kong, China \\ * Correspondence to: \\ Dr. Gary Tse PhD FRCP, FFPH \\ Tianjin Key Laboratory of Ionic-Molecular Function of Cardiovascular Disease, Department of \\ Cardiology, Tianjin Institute of Cardiology, Second Hospital of Tianjin Medical University, Tianjin \\ 300211, China \\ Email: garytse86@gmail.com
}

\section{Prof. Bernard Man Yung Cheung MB BChir PhD FRCP}

Department of Medicine, The University of Hong Kong, Room 405B, 4/F, Professorial Block, Queen Mary Hospital, 102 Pok Fu Lam Road, Pok Fu Lam,

Hong Kong, China

Email: mycheung@hku.hk

Tel: +85222554347 
Dr. Qingpeng Zhang PhD

School of Data Science, City University of Hong Kong,

Hong Kong SAR, China

Email: qingpeng.zhang@cityu.edu.hk

$+85234424727$

\section{Key Points:}

- We report that an association between ACEIs/ARBs with COVID-19 related mortality even after adjusting for cardiovascular and other comorbidities, as well as medication use.

- Patients with greater comorbidity burden and laboratory markers reflecting deranged clotting, renal and liver function, and increased tissue inflammation, and ACEI/ARB use have a higher mortality risk.

Key words: ACE inhibitors; angiotensin receptor blockers; COVID-19; mortality

\section{Acknowledgements: None}

Conflicits of Interest: None 


\begin{abstract}
Aims: Renin-angiotensin system blockers such as angiotensin-converting enzyme inhibitors (ACEIs) and angiotensin receptor blockers (ARBs) may increase the risk of adverse outcomes in COVID-19. In this study, the relationships between ACEI/ARB use and COVID-19 related mortality were examined.
\end{abstract}

Methods: Consecutive patients diagnosed with COVID-19 by RT-PCR at the Hong Kong Hospital Authority between $1^{\text {st }}$ January and $28^{\text {th }}$ July 2020 were included.

Results: This study included 2774 patients. The mortality rate of the COVID-19 positive group was $1.5 \%(\mathrm{n}=42)$. Those who died had a higher median age (82.3[76.5-89.5] vs. 42.9[28.2-59.5] years old; $\mathrm{P}<0.0001$ ), more likely to have baseline comorbidities of cardiovascular disease, diabetes mellitus, hypertension, and chronic kidney disease $(\mathrm{P}<0.0001)$. They were more frequently prescribed ACEI/ARBs at baseline, and steroids, lopinavir/ritonavir, ribavirin and hydroxychloroquine during admission $(\mathrm{P}<0.0001)$. They also had a higher white cell count, higher neutrophil count, lower platelet count, prolonged prothrombin time and activated partial thromboplastin time, higher D-dimer, troponin, lactate dehydrogenase, creatinine, alanine transaminase, aspartate transaminase and alkaline phosphatase $(\mathrm{P}<0.0001)$. Multivariate Cox regression showed that age, cardiovascular disease, renal disease, diabetes mellitus, the use of ACEIs/ARBs and diuretics, and various laboratory tests remained significant predictors of mortality.

Conclusions: We report that an association between ACEIs/ARBs with COVID-19 related mortality even after adjusting for cardiovascular and other comorbidities, as well as medication use. Patients 
with greater comorbidity burden and laboratory markers reflecting deranged clotting, renal and liver function, and increased tissue inflammation, and ACEI/ARB use have a higher mortality risk.

\section{Introduction}

The use of angiotensin $\square$ converting enzyme (ACE) inhibitors and angiotensin receptor blockers (ARBs) has been associated with poor disease outcomes in coronavirus disease 2019 (COVID-19). For example, a population-based cohort study from Korea found that ACE-I or ARB therapy in patients with severe COVID-19 was associated with the occurrence of severe complications and increased in-hospital mortality [1]. By contrast, other studies did not identify an increased risk of severe COVID-19 [2, 3] or mortality [4] among patients taking ACEIs or ARBs. A systematic review and meta-analysis of 33 studies found that ACE inhibitor use was marker of increased mortality risk in some, but not all, COVID-19 disease settings [5], whilst another found that The risk of mortality and severe outcomes are also unchanged among COVID-19 patients taking ACEI/ARB [6]. Nevertheless, prior use of RAAS inhibitors was associated with lower risk mortality from COVID-19 specifically in patients with hypertension [7]. Given these conflicting findings, we examined whether the use of ACEIs/ARBs is related to COVID-19 mortality using territory-wide data from Hong Kong.

\section{Methods}

Study design and population 
medRxiv preprint doi: https://doi.org/10.1101/2020.12.21.20248645; this version posted December 23, 2020. The copyright holder for this preprint (which was not certified by peer review) is the author/funder, who has granted medRxiv a license to display the preprint in perpetuity. It is made available under a CC-BY 4.0 International license .

This study was approved by the Institutional Review Board of the University of Hong Kong/Hospital Authority Hong Kong West Cluster. This was a retrospective, territory-wide cohort study of patients undergoing COVID-19 RT-PCR testing between $1^{\text {st }}$ January and $28^{\text {th }}$ July 2020. The patients were identified from the Clinical Data Analysis and Reporting System (CDARS), a territory-wide database that centralizes patient information from individual local hospitals to establish comprehensive medical data, including clinical characteristics, disease diagnosis, laboratory results, and drug treatment details. The system has been previously used by both our team and other teams in Hong Kong [8-11]. The list of conditions identified is detailed in the Supplementary Appendix. Patients and the public were not engaged in this study.

\section{Outcomes and statistical analysis}

All-cause mortality was the primary outcome for the COVID-19 positive group, with last date of mortality status on $8^{\text {th }}$ August 2020. Continuous variables were presented as median $(95 \%$ confidence interval $[\mathrm{CI}]$ or interquartile range $[\mathrm{IQR}]$ ) and categorical variables were presented as count $(\%)$. The Mann- Whitney U test was used to compare continuous variables. The $\chi^{2}$ test with Yates' correction was used for $2 \times 2$ contingency data. Statistical analyses were performed using RStudio software (Version: 1.1.456) and Python (Version: 3.6).

\section{Results}


A total of 2774 patients with confirmed COVID-19 were included. Their baseline characteristics are shown in Table 1. A total of 42 deaths occurred (1.5\%) in the 2774 patients who were diagnosed with COVID-19. Those who died had a higher median age (82.3[76.5-89.5] vs. 42.9[28.2-59.5] years old), more likely to have baseline comorbidities of cardiovascular disease, diabetes mellitus, hypertension, and chronic kidney disease. Moreover, they were more frequently prescribed ACEI/ARBs at baseline, and steroids, lopinavir/ritonavir, ribavirin and hydroxychloroquine during admission. Finally, patients who died had a higher white cell count, higher neutrophil count, lower platelet count, prolonged PT and activated partial thromboplastin time, higher D-dimer, troponin and lactate dehydrogenase. Moreover, creatinine, alanine transaminase, aspartate transaminase and alkaline phosphatase were significantly higher for the mortality group.

Univariate Cox regression was used to identify predictors of mortality in patients who were positive for COVID-19 (Table 2). This identified age, baseline cardiovascular and renal diseases, diabetes, hypertension, the use of ACEIs/ARBs, other anti-hypertensive agents, and anti-viral drugs in COVID-19, as well as various haematology and biochemical tests. On multivariate analysis, age, cardiovascular disease, renal disease, diabetes mellitus, the use of ACEIs/ARBs and diuretics, and various laboratory tests remained significant predictors of mortality (Table 3).

\section{Discussion}

The main findings of this territory-wide cohort study are that 1) a higher proportion of ACEI/ARB prescription was observed for the COVID-19 positive compared to COVID-19 negative 
group and 2) those who died had a higher median age and more likely to have baseline comorbidities of cardiovascular disease, diabetes mellitus, hypertension, and chronic kidney disease and 3) ACEI/ARB use was significantly associated with mortality even after adjusting for cardiovascular comorbidities and the use of other anti-hypertensive agents. Moreover, they were more frequently prescribed ACEI/ARBs at baseline, and steroids, lopinavir/ritonavir, ribavirin and hydroxychloroquine during admission.

The relationship between ACEI/ARB use and disease outcomes in COVID-19 has been investigated. Many of the previous studies have included hospitalized patients only. For example, in a multi-centre study from China, it was found that in hospitalized patients with COVID-19 and existing hypertension, ACEI/ARB inpatient users had a lower risk of all-cause mortality compared with nonusers [12]. In our study, we found that in those who died, there was a higher frequency of patients with ACE inhibitor/ARB use compared to those who remained alive. However, on multivariate Cox regression, ACEI/ARB use retained its significance for mortality prediction even after multivariate adjustment for cardiovascular comorbidities and the use of other anti-hypertensive agents. Nevertheless, the frequency of hypertension, diabetes mellitus and cardiovascular disease were also higher in the mortality group. This is in keeping with previous demonstrations that patients with pre-existing cardio-metabolic comorbidities are at higher risk of severe disease or mortality [13].

In terms of laboratory findings, prior studies have demonstrated the presence of organ damage in COVID-19. Indeed, there have been reported associations between elevations in myocardial biomarkers and mortality [14-16]. Alterations in other laboratory markers that have been associated 
with adverse outcomes in COVID-19 include increases in D-dimer levels [17], liver enzymes [18], neutrophil count [19], activated partial thromboplastin time and prothrombin time [20]. In our study, we also found that patients who died had a higher white cell count, higher neutrophil count, lower platelet count, prolonged prothrombin time and activated partial thromboplastin time, D-dimer, troponin, lactate dehydrogenase, creatinine, alanine transaminase, aspartate transaminase and alkaline phosphatase.

\section{Conclusion}

Taken together, we report that an association between ACEIs or ARBs with COVID-19 related mortality even after adjusting for cardiovascular and other comorbidities, as well as medication use. Our findings suggest that patients with greater comorbidity burden and laboratory markers reflecting deranged clotting, renal and liver function, and increased tissue inflammation, as well as ACEI/ARB use have a higher mortality risk.

\section{Conflicts of Interest}

None.

\section{Funding}

None. 


\section{Contributorship Statement}

GT: study conception, study design, data acquisition, statistical analysis, data interpretation, manuscript drafting, critical revision of manuscript

JZ: statistical analysis, data interpretation, manuscript drafting, critical revision of manuscript

SL, WTW, XW, WKKW, TL, ZC, DZ, KCKW: data interpretation, critical revision of manuscript

BMYC, QZ: project administration and supervision, statistical analysis, data interpretation, critical revision of manuscript

Guarantor of overall content: GT

\section{References}

1. Lim JH, Cho JH, Jeon $Y$, Kim JH, Lee GY, Jeon S, et al. Adverse impact of renin-angiotensin system blockade on the clinical course in hospitalized patients with severe COVID-19: a retrospective cohort study. Sci Rep. 2020 Nov 20;10(1):20250.

2. Bauer AZ, Gore R, Sama SR, Rosiello R, Garber L, Sundaresan D, et al. Hypertension, medications, and risk of severe COVID-19: A Massachusetts community-based observational study. J Clin Hypertens (Greenwich). 2020 Nov 21.

3. Martinez-Del Rio J, Piqueras-Flores J, Nieto-Sandoval Martin de la Sierra P, Negreira-Caamano M, Aguila-Gordo D, Mateo-Gomez C, et al. Comparative analysis between the use of renin-angiotensin system antagonists and clinical outcomes of hospitalized patients with COVID-19 respiratory infection. Med Clin (Engl Ed). 2020 Nov 12. 
4. Braude P, Carter B, Short R, Vilches-Moraga A, Verduri A, Pearce L, et al. The influence of ACE inhibitors and ARBs on hospital length of stay and survival in people with COVID-19. Int J Cardiol Heart Vasc. 2020 Dec;31:100660.

5. Nunes JPL. Mortality and use of angiotensin-converting enzyme inhibitors in COVID 19 disease: a systematic review. Porto Biomed J. 2020 Nov-Dec;5(6):e085.

6. Xu J, Teng Y, Shang L, Gu X, Fan G, Chen Y, et al. The Effect of Prior ACEI/ARB Treatment on COVID-19 Susceptibility and Outcome: A Systematic Review and Meta-Analysis. Clin Infect Dis. 2020 Oct 20.

7. Ssentongo AE, Ssentongo P, Heilbrunn ES, Lekoubou A, Du P, Liao D, et al. Renin-angiotensin-aldosterone system inhibitors and the risk of mortality in patients with hypertension hospitalised for COVID-19: systematic review and meta-analysis. Open Heart. 2020 Nov;7(2).

8. Li CK, Xu Z, Ho J, Lakhani I, Liu YZ, Bazoukis G, et al. Association of NPAC score with survival after acute myocardial infarction. Atherosclerosis. 2020 May;301:30-6.

9. Ju C, Lai RWC, Li KHC, Hung JKF, Lai JCL, Ho J, et al. Comparative cardiovascular risk in users versus non-users of xanthine oxidase inhibitors and febuxostat versus allopurinol users. Rheumatology (Oxford). 2019 Dec 24.

10. Lau WCY, Cheung CL, Man KKC, Chan EW, Sing CW, Lip GYH, et al. Association Between Treatment With Apixaban, Dabigatran, Rivaroxaban, or Warfarin and Risk for Osteoporotic Fractures Among Patients With Atrial Fibrillation. Ann Intern Med. 2020 May 19. 
11. Lau WC, Chan EW, Cheung CL, Sing CW, Man KK, Lip GY, et al. Association Between

Dabigatran vs Warfarin and Risk of Osteoporotic Fractures Among Patients With

Nonvalvular Atrial Fibrillation. JAMA. 2017 Mar 21;317(11):1151-8.

12. Zhang P, Zhu L, Cai J, Lei F, Qin JJ, Xie J, et al. Association of Inpatient Use of

Angiotensin-Converting Enzyme Inhibitors and Angiotensin II Receptor Blockers With

Mortality Among Patients With Hypertension Hospitalized With COVID-19. Circ Res. 2020

Jun 5;126(12):1671-81.

13. Li X, Guan B, Su T, Liu W, Chen M, Bin Waleed K, et al. Impact of cardiovascular

disease and cardiac injury on in-hospital mortality in patients with COVID-19: a systematic

review and meta-analysis. Heart. 2020 Aug;106(15):1142-7.

14. Guo T, Fan Y, Chen M, Wu X, Zhang L, He T, et al. Cardiovascular Implications of Fatal

Outcomes of Patients With Coronavirus Disease 2019 (COVID-19). JAMA Cardiol. 2020

Mar 27.

15. Shi S, Qin M, Shen B, Cai Y, Liu T, Yang F, et al. Association of Cardiac Injury With Mortality in Hospitalized Patients With COVID-19 in Wuhan, China. JAMA Cardiol. 2020 Mar 25.

16. Wang Y, Roever L, Tse G, Liu T. 2019-Novel Coronavirus-Related Acute Cardiac Injury Cannot Be Ignored. Curr Atheroscler Rep. 2020 May 7;22(3):14.

17. Yao Y, Cao J, Wang Q, Shi Q, Liu K, Luo Z, et al. D-dimer as a biomarker for disease severity and mortality in COVID-19 patients: a case control study. Journal of Intensive Care. 2020 2020/07/10;8(1):49. 
medRxiv preprint doi: https://doi.org/10.1101/2020.12.21.20248645; this version posted December 23, 2020. The copyright holder for this preprint (which was not certified by peer review) is the author/funder, who has granted medRxiv a license to display the preprint in perpetuity. It is made available under a CC-BY 4.0 International license .

18. Cai Q, Huang D, Yu H, Zhu Z, Xia Z, Su Y, et al. COVID-19: Abnormal liver function tests. J Hepatol. 2020 Sep;73(3):566-74.

19. Huang C, Wang Y, Li X, Ren L, Zhao J, Hu Y, et al. Clinical features of patients infected with 2019 novel coronavirus in Wuhan, China. Lancet. 2020 Feb 15;395(10223):497-506. 20. Tang N, Li D, Wang X, Sun Z. Abnormal coagulation parameters are associated with poor prognosis in patients with novel coronavirus pneumonia. J Thromb Haemost. 2020 Apr;18(4):844-7. 
Table 1. Demographic, epidemiological, clinical characteristics and laboratory details of patients who were tested positive for COVID-19 in Hong Kong up to and including $28^{\text {th }}$ July 2020. Patients were stratified by mortality status with latest available death information on $5^{\text {th }}$ August 2020 .

COVID-19 = coronavirus disease 2019; APTT = Activated partial thromboplastin time; IQR = Interquartile range; $*$ for $\mathrm{p} \leq 0.05, * *$ for $\mathrm{p} \leq 0.01, * * *$ for $\mathrm{p} \leq 0.001$

\begin{tabular}{|c|c|c|c|c|}
\hline Characteristics & $\begin{array}{l}\text { All COVID-19 patients }(n=2774) \\
\text { Median (IQR) or count }(\%)\end{array}$ & $\begin{array}{l}\text { Death }(n=42) \\
\text { Median }(I Q R) \text { or count }(\%)\end{array}$ & $\begin{array}{l}\text { Alive }(n=2732) \\
\text { Median (IQR) or count }(\%)\end{array}$ & P value \\
\hline \multicolumn{5}{|l|}{ Demographics } \\
\hline Age, year,[IQR] max & $43.54[28.61-60.00] 97.51$ & $82.59[76.51,89.54] 95.59$ & $42.885[28.24,59.47] 97.51$ & $0.0031 * *$ \\
\hline \multicolumn{5}{|l|}{ Sex } \\
\hline Male & $1369(49.35 \%)$ & $26(61.90 \%)$ & $1343(49.16 \%)$ & $<0.0001 * * *$ \\
\hline Female & $1405(50.65 \%)$ & $16(38.10 \%)$ & $1389(50.84 \%)$ & $<0.0001 * * *$ \\
\hline \multicolumn{5}{|l|}{ Comorbidities } \\
\hline Cardiovascular & $196(7.07 \%)$ & $20(47.62 \%)$ & $176(6.44 \%)$ & $<0.0001 * * *$ \\
\hline Respiratory & $2145(77.33 \%)$ & $36(85.71 \%)$ & $2109(77.20 \%)$ & $0.0122 *$ \\
\hline Kidney & $133(4.79 \%)$ & $15(35.71 \%)$ & $118(4.32 \%)$ & $<0.0001 * * *$ \\
\hline Endocrine & $29(1.05 \%)$ & $1(2.38 \%)$ & $28(1.02 \%)$ & $0.0013 * *$ \\
\hline Diabetes & $294(10.60 \%)$ & $18(42.86 \%)$ & $276(10.10 \%)$ & $<0.0001 * * *$ \\
\hline Hypertension & $413(14.89 \%)$ & $24(57.14 \%)$ & $389(14.24 \%)$ & $<0.0001 * * *$ \\
\hline Gastrointestinal & $2127(76.68 \%)$ & $32(76.19 \%)$ & $2095(76.68 \%)$ & 0.212 \\
\hline \multicolumn{5}{|l|}{ Drugs } \\
\hline ACEI/ARB (baseline) & $279(10.06 \%)$ & $16(38.10 \%)$ & $263(9.63 \%)$ & $<0.0001 * * *$ \\
\hline Steroids & $143(5.16 \%)$ & $6(14.29 \%)$ & $137(5.01 \%)$ & $<0.0001 * * *$ \\
\hline Lopinavir/Ritonavir (Kaletra) & $29(1.05 \%)$ & $4(9.52 \%)$ & $25(0.92 \%)$ & 0.355 \\
\hline Ribavirin & $519(18.71 \%)$ & $2(4.76 \%)$ & $517(18.92 \%)$ & $<0.0001 * * *$ \\
\hline
\end{tabular}




\begin{tabular}{|c|c|c|c|c|}
\hline Interferon beta & $317(11.43 \%)$ & $3(7.14 \%)$ & $314(11.49 \%)$ & $<0.0001 * * *$ \\
\hline Hydroxychloroquine & $38(1.37 \%)$ & $4(9.52 \%)$ & $34(1.24 \%)$ & $0.0126^{*}$ \\
\hline Calcium channel blockers & $436(15.72 \%)$ & $26(61.90 \%)$ & $410(15.01 \%)$ & $0.0233 *$ \\
\hline Beta blockers & $182(6.56 \%)$ & $12(28.57 \%)$ & $170(6.22 \%)$ & $0.0015 * *$ \\
\hline Diuretics for heart failure & $13(0.47 \%)$ & $2(4.76 \%)$ & $11(0.40 \%)$ & $0.0016 * *$ \\
\hline Diuretics for hypertension & $8(0.29 \%)$ & $1(2.38 \%)$ & $7(0.26 \%)$ & $0.0031 * *$ \\
\hline Nitrates & $25(0.90 \%)$ & $1(2.38 \%)$ & $24(0.88 \%)$ & $0.0121 *$ \\
\hline Other Antihypertensive drugs & $20(0.72 \%)$ & $4(9.52 \%)$ & $16(0.59 \%)$ & 0.1351 \\
\hline \multicolumn{5}{|l|}{ Complete blood counts } \\
\hline $\mathrm{MCV}, \mathrm{fL}$ & $86.4(82.5-90.0) ; n=1609$ & $90.1(86.7-94.4) ; n=35$ & 86.4(82.4-89.9); $n=1574$ & $0.0023 * *$ \\
\hline Basophil, x10^9/L & $0.01(0.0-0.03) ; \mathrm{n}=2373$ & $0.02(0.004-0.04) ; n=37$ & $0.01(0.0-0.03) ; \mathrm{n}=2336$ & 0.116 \\
\hline Eosinophil, x10^9/L & $0.1(0.01-0.17) ; \mathrm{n}=2501$ & $0.01(0.0-0.1) ; \mathrm{n}=41$ & $0.1(0.01-0.17) ; n=2460$ & 0.0921 \\
\hline Lymphocyte, x10^9/L & $1.6(1.2-2.1) ; \mathrm{n}=2511$ & $1.06(0.7-1.61) ; \mathrm{n}=41$ & $1.6(1.2-2.1) ; \mathrm{n}=2470$ & $<0.0001 * * *$ \\
\hline Metamyelocyte, x10^9/L & $0.17(0.09-0.645) ; n=4$ & $0.07(0.07-0.07) ; \mathrm{n}=1$ & $0.23(0.11-1.06) ; \mathrm{n}=3$ & $<0.0001 * * *$ \\
\hline Monocyte, x10^9/L & $0.5(0.38-0.61) ; \mathrm{n}=2511$ & $0.6(0.43-0.7) ; n=41$ & $0.5(0.38-0.61) ; n=2470$ & $0.0422 *$ \\
\hline Neutrophil, x10^9/L & $3.4(2.53-4.5) ; n=2511$ & $6.69(4.5-11.61) ; n=41$ & $3.37(2.5-4.47) ; n=2470$ & $<0.0001 * * *$ \\
\hline WBC, $\times 10^{\wedge} 9 / \mathrm{L}$ & $5.8(4.7-7.285) ; n=2589$ & $7.87(6.48-12.16) ; n=41$ & $5.79(4.7-7.2) ; n=2548$ & $0.0321 *$ \\
\hline $\mathrm{MCH}, \mathrm{g} / \mathrm{dL}$ & $33.8(33.0-34.5) ; n=2589$ & $33.6(32.2-34.1) ; n=41$ & $33.8(33.0-34.5) ; \mathrm{n}=2548$ & 0.0621 \\
\hline Myelocyte, $x 10^{\wedge} 9 / \mathrm{L}$ & $0.29(0.11-0.4) ; \mathrm{n}=11$ & $0.175(0.175-0.175) ; \mathrm{n}=2$ & $0.35(0.12-0.48) ; \mathrm{n}=9$ & $<0.0001 * * *$ \\
\hline Platelet, $x 10^{\wedge} 9 / \mathrm{L}$ & $237.0(180.0-298.0) ; \mathrm{n}=2589$ & $197.0(138.0-256.0) ; \mathrm{n}=41$ & $234.0(175.0-297.0) ; \mathrm{n}=2548$ & $<0.0001 * * *$ \\
\hline Reticulocyte, x10^9/L & $44.15(27.85-58.15) ; n=20$ & $42.8(42.8-42.8) ; n=2$ & $45.0(26.5-63.2) ; n=18$ & $<0.0001 * * *$ \\
\hline $\mathrm{RBC}, \mathrm{x} 10^{\wedge} 12 / \mathrm{L}$ & $4.61(4.235-5.02) ; n=2589$ & $3.97(3.23-4.38) ; n=41$ & $4.62(4.25-5.02) ; n=2548$ & 0.0721 \\
\hline
\end{tabular}

Biochemical tests 


\section{Sodium, mmol/L}

Potassium, mmol/L

Urea, $\mathrm{mmol} / \mathrm{L}$

Creatinine, umol/L

Albumin, g/L

Protein, g/L

Urate, $\mathrm{mmol} / \mathrm{L}$

Alkaline Phosphatase, U/L

Aspartate Transaminase, U/L

Alanine Transaminase, U/L

Bilirubin, $\mu \mathrm{mol} / \mathrm{L}$
139.0(137.0-140.2); $\mathrm{n}=2591$

3.8(2.6-3.78); $\mathrm{n}=2590$

4.0(3.14-5.0); $\mathrm{n}=2590$

68.0(57.0-82.0); $\mathrm{n}=2591$

40.0(35.3-43.8); $\mathrm{n}=2588$

73.1(69.0-77.0); $\mathrm{n}=2323$

$0.3038(0.246-0.4041) ; n=111$

64.4(54.0-80.0); $\mathrm{n}=2587$

26.0(20.0-39.0); $\mathrm{n}=870$

$24.0(16.0-39.0) ; n=2091$

8.0(5.8-12.0); $\mathrm{n}=2587$
137.2(133.2-144.155); $\mathrm{n}=41$

4.4(3.87-4.79); $\mathrm{n}=41$

12.5(7.25-21.61); $\mathrm{n}=41$

137.0(82.5-196.8); $\mathrm{n}=41$

25.0(20.05-30.3); $\mathrm{n}=41$

66.1(60.8-72.0); $n=31$

$0.32(0.292-0.62) ; n=3$

84.0(68.0-117.0); $\mathrm{n}=41$

92.5(23.0-225.0); $\mathrm{n}=18$

47.5(25.9-78.0); $\mathrm{n}=34$

10.1(6.65-29.65); $\mathrm{n}=41$
139.0(137.0-140.1); $\mathrm{n}=2550$

4.01(4.275-3.9); $n=2549$

3.9(3.1-4.93); $\mathrm{n}=2549$

40.0(35.1-43.8); $\mathrm{n}=2547$

73.4(69.6-77.0); $\mathrm{n}=2292$

$0.3019(0.243-0.4019) ; n=108$

64.0(54.0-79.0); $\mathrm{n}=2546$

23.0(16.0-38.0); $\mathrm{n}=2057$

8.0(5.7-12.0); $\mathrm{n}=2546$
68.0(57.0-81.0); $\mathrm{n}=2550$

26.0(20.0-38.0); $\mathrm{n}=852$

0.318

0.217

$<0.0001 * * *$

$<0.0001 * * *$

0.4323

$<0.0001 * * *$

0.3622

$<0.0001 * * *$

$<0.0001 * * *$

$<0.0001 * * *$

$<0.0001 * * *$

\section{Cardiac function tests and clotting}

High sensitive troponin-I, ng/L

290.0(190.0-585.0); $\mathrm{n}=252$

3.0(1.2-6.19); $\mathrm{n}=499$

Lactate dehydrogenase, U/L

175.0(150.0-201.0); $\mathrm{n}=1003$

11.9(11.4-12.5); $\mathrm{n}=367$

$30.55(27.4-34.45) ; \mathrm{n}=518$

13.4(12.4-14.5); $\mathrm{n}=2589$

4.4(3.9-4.24); $\mathrm{n}=2038$

$2.1(6.5-3.4) ; n=391$

3278.59(3279.0-3279.0); $\mathrm{n}=1$

$22.9(13.0-37.0) ; \mathrm{n}=9$

364.0(306.0-623.0); $\mathrm{n}=11$

13.7(13.45-15.1); $n=3$

31.9(30.2-33.6); $\mathrm{n}=5$

\section{Diabetes mellitus tests}

HbA1c, g/dL

Glucose, mmol/L

Cholesterol, mmol/L
$11.6(9.2-13.0) ; \mathrm{n}=41$

7.9(6.01-8.9); $\mathrm{n}=37$

2.0(1.2-3.5); $\mathrm{n}=15$
290.0(190.0-580.0); $\mathrm{n}=251$

$3.0(1.2-5.58) ; \mathrm{n}=490$

174.0(149.0-200.0); $\mathrm{n}=992$

11.9(11.4-12.5); $n=364$

30.5(27.4-34.5); $\mathrm{n}=513$

13.4(12.4-14.5); $\mathrm{n}=2548$

8.8(3.2-7.6); $\mathrm{n}=2001$

$0.6(0.04-2.8) ; n=376$
$<0.0001 * * *$

$<0.0001 * * *$

$<0.0001 * * *$

$<0.0001 * * *$

0.1309

$$
\begin{aligned}
& <0.0001 * \\
& * * 0.0035 * * \\
& <0.0001 *
\end{aligned}
$$$$
\text { ** }
$$ 
Table 2. Univariate Cox regression to predict mortality after COVID-19 presentation ( $\mathrm{n}=2774)$

\begin{tabular}{|c|c|c|c|}
\hline Variable & Hazard ratio $(95 \% \mathrm{CI})$ & $Z$ value & P value \\
\hline Sex & $1.612[0.8605,3.019]$ & 1.491 & 0.136 \\
\hline Age & $1.132[1.104,1.161]$ & 9.771 & $<0.0001 * * *$ \\
\hline \multicolumn{4}{|l|}{ Comorbidities } \\
\hline Cardiovascular & $13.25[7.181,24.44]$ & 8.269 & $<0.0001 * * *$ \\
\hline Respiratory & $1.723[0.7249,4.097]$ & 1.232 & 0.218 \\
\hline Kidney & $10.95[5.744,20.89]$ & 7.267 & $<0.0001 * * *$ \\
\hline Endocrine & $2.423[0.3331,17.63]$ & 0.874 & 0.382 \\
\hline Diabetes mellitus & $6.81[3.675,12.62]$ & 6.096 & $<0.0001 * * *$ \\
\hline Hypertension & 7.498[4.046,13.89] & 6.402 & $<0.0001 * * *$ \\
\hline Gastrointestinal & $0.9448[0.4632,1.927]$ & -0.156 & 0.876 \\
\hline \multicolumn{4}{|l|}{ Drugs } \\
\hline ACEI/ARB (baseline) & $5.87[3.13,11]$ & 5.53 & $<0.0001 * * *$ \\
\hline Steroids & $1.21[0.46,2.14]$ & 2.005 & 0.0996 \\
\hline Lopinavir/Ritonavir (Kaletra) & $11.64[4.15,32.67]$ & 4.663 & $<0.0001 * * *$ \\
\hline Ribavirin & $0.22[0.054,0.919]$ & -2.077 & $0.0378 *$ \\
\hline Interferon beta & $0.613[0.1892,1.986]$ & -0.816 & 0.415 \\
\hline Hydroxychloroquine & $1.04[0.45,2.11]$ & -0.006 & 0.0995 \\
\hline Calcium channel blockers & $2.601[1.592,3.11]$ & 6.721 & $0.0036 * *$ \\
\hline Beta blockers & $3.113[2.119,4.98]$ & 5.274 & $0.0065 * *$ \\
\hline Diuretics for heart failure & $12.17[2.938,50.4]$ & 3.446 & $0.0006^{* * *}$ \\
\hline Diuretics for hypertension & $9.218[1.267,67.06]$ & 2.194 & $0.0282 *$ \\
\hline Nitrates & $2.773[0.3812,20.17]$ & 1.007 & 0.3145 \\
\hline Other Antihypertensive drugs & $6.61[5.92,46.62]$ & 5.338 & $0.0112 *$ \\
\hline \multicolumn{4}{|l|}{ Complete blood counts } \\
\hline $\mathrm{MCV}, \mathrm{fL}$ & $1.109[1.047,1.174]$ & 3.534 & $<0.0001 * * *$ \\
\hline
\end{tabular}




\begin{tabular}{|c|c|c|c|}
\hline Basophil, x10^9/L & $95.95[1.414,641]$ & 2.037 & $0.0417 *$ \\
\hline Eosinophil, $\times 10^{\wedge} 9 / \mathrm{L}$ & $1.972[0.3964,9.809]$ & 0.829 & 0.407 \\
\hline Lymphocyte, x10^9/L & $0.31[0.173,0.549]$ & -3.997 & $<0.0001 * * *$ \\
\hline Metamyelocyte, $\times 10^{\wedge} 9 / \mathrm{L}$ & $1.113[0.7615,2.145]$ & -0.004 & $0.034 *$ \\
\hline Monocyte, $\mathrm{x} 10^{\wedge} 9 / \mathrm{L}$ & $6.308[2.838,14.02]$ & 4.519 & $<0.0001 * * *$ \\
\hline Neutrophil, x10^9/L & $1.273[1.227,1.321]$ & 12.71 & $<0.0001 * * *$ \\
\hline White blood count, $x 10^{\wedge} 9 / \mathrm{L}$ & $1.053[1.034,1.073]$ & 5.58 & $<0.0001 * * *$ \\
\hline $\mathrm{MCH}, \mathrm{g} / \mathrm{dL}$ & $0.661[0.539,0.811]$ & -3.962 & $<0.0001 * * *$ \\
\hline Myelocyte, x10^9/L & $1.21[0.15,2.9]$ & -0.925 & $0.0351 *$ \\
\hline Platelet, $\times 10^{\wedge} 9 / \mathrm{L}$ & $0.9891[0.9843,0.9941]$ & -4.318 & $<0.0001 * * *$ \\
\hline Reticulocyte, $\times 10^{\wedge} 9 / \mathrm{L}$ & $0.9915[0.9504,1.034]$ & -0.396 & 0.692 \\
\hline Red blood count, $\times 10^{\wedge} 12 / \mathrm{L}$ & $0.1905[0.1259,0.2883]$ & -7.847 & $<0.0001 * * *$ \\
\hline \multicolumn{4}{|l|}{ Biochemical tests } \\
\hline Sodium, mmol/L & $1.012[0.9188,1.114]$ & 0.233 & 0.816 \\
\hline Potassium, mmol/L & $4.508[3.357,6.053]$ & 10.02 & $<0.0001 * * *$ \\
\hline Urea, mmol/L & $1.169[1.145,1.193]$ & 15.15 & $<0.0001 * * *$ \\
\hline Creatinine, umol/L & $1.005[1.004,1.006]$ & 9.95 & $<0.0001 * * *$ \\
\hline Albumin, $\mathrm{g} / \mathrm{L}$ & $0.81[0.79,0.84]$ & -12.78 & $<0.0001 * * *$ \\
\hline Protein, g/L & $0.8757[0.8431,0.9096]$ & -6.85 & $<0.0001 * * *$ \\
\hline Urate, $\mathrm{mmol} / \mathrm{L}$ & $151.4[0.03189,718363]$ & 1.162 & 0.245 \\
\hline Alkaline Phosphatase, U/L & $1.003[1.002,1.004]$ & 4.727 & $<0.0001 * * *$ \\
\hline Aspartate Transaminase, U/L & $1.002[1.001,1.002]$ & 7.48 & $<0.0001 * * *$ \\
\hline Alanine Transaminase, U/L & $1.013[1.01,1.016]$ & 7.338 & $<0.0001 * * *$ \\
\hline Bilirubin, $\mu \mathrm{mol} / \mathrm{L}$ & $1.00[0.9999,1.001]$ & 9.114 & $<0.0001 * * *$ \\
\hline \multicolumn{4}{|c|}{ Cardiac function tests and clotting } \\
\hline D-dimer, ng/mL & $1.00[0.9994,1.001]$ & 1.669 & 0.0951 \\
\hline High sensitive troponin-I, ng/L & $1.005[1.004,1.006]$ & -0.097 & 0.923 \\
\hline
\end{tabular}




\begin{tabular}{|c|c|c|c|}
\hline Lactate dehydrogenase, U/L & $1.079[0.8284,1.406]$ & 7.777 & $<0.0001 * * *$ \\
\hline Prothrombin time, sec & $1.41[1.116,1.78]$ & 2.885 & $0.00391 * *$ \\
\hline APTT, sec & $1.035[0.8662,1.236]$ & 0.377 & 0.706 \\
\hline \multicolumn{4}{|c|}{ Diabetes mellitus and lipid tests } \\
\hline $\mathrm{HbA} 1 \mathrm{c}, \mathrm{g} / \mathrm{dL}$ & $0.5701[0.493,0.6593]$ & -7.579 & $<0.0001 * * *$ \\
\hline Glucose, $\mathrm{mmol} / \mathrm{L}$ & $1.164[1.103,1.229]$ & 5.528 & $<0.0001 * * *$ \\
\hline Cholesterol, $\mathrm{mmol} / \mathrm{L}$ & $0.6611[0.4159,1.051]$ & -1.749 & 0.0802 \\
\hline Triglycerides, mmol/L & $0.9896[0.6185,1.583]$ & -0.044 & 0.965 \\
\hline
\end{tabular}

Triglycerides, $\mathrm{mmol} / \mathrm{L}$

$0.9896[0.6185,1.583]$ 
Table 3. Multivariate Cox regression to predict mortality after COVID-19 presentation with significant univariate predictors as input ( $\mathrm{n}=2774$ )

\begin{tabular}{|c|c|c|c|}
\hline Variable & Hazard ratio $(95 \% \mathrm{CI})$ & $Z$ value & $P$ value \\
\hline \multicolumn{4}{|l|}{ Demographics } \\
\hline Age & $1.13[1.09,1.20]$ & 4.37 & $<0.0001 * * *$ \\
\hline \multicolumn{4}{|l|}{ Comorbidities } \\
\hline Cardiovascular & $1.12[0.47,2.66]$ & 1.28 & $<0.0001 * * *$ \\
\hline Kidney & $1.25[0.42,2.88]$ & 2.14 & $0.0032 * *$ \\
\hline Diabetes mellitus & $1.81[0.77,4.36]$ & 2.22 & $0.0412 *$ \\
\hline Hypertension & $0.84[0.36,1.99]$ & -0.41 & 0.856 \\
\hline \multicolumn{4}{|l|}{ Drugs } \\
\hline ACEI/ARB (baseline) & $1.24[0.33,2.78]$ & 1.16 & $<0.0001 * * *$ \\
\hline Lopinavir/Ritonavir (Kaletra) & $83.96[0.76,103.11]$ & 3.68 & $<0.0001 * * *$ \\
\hline Antihypertensive drugs & $1.76[1.31,9.21]$ & -0.28 & 0.322 \\
\hline Diuretics for heart failure & $16.12[2.13,121.21]$ & 28.95 & $<0.0001 * * *$ \\
\hline \multicolumn{4}{|l|}{ Complete blood counts } \\
\hline $\mathrm{MCV}, \mathrm{fL}$ & $1.03[0.95,1.06]$ & 0.72 & 0.1801 \\
\hline Monocyte, x10^9/L & $0.70[0.14,3.40]$ & -0.47 & 0.3411 \\
\hline Neutrophil, x10^9/L & $1.10[1.01,1.22]$ & 2.13 & $0.0037 * *$ \\
\hline White blood count, $x 10^{\wedge} 9 / \mathrm{L}$ & $1.03[0.90,1.15]$ & 0.16 & 0.2633 \\
\hline \multicolumn{4}{|l|}{ Biochemical tests } \\
\hline Potassium, mmol/L & $1.53[0.50,4.68]$ & 0.75 & 0.1734 \\
\hline Urea, mmol/L & $1.15[1.07,1.22]$ & 3.72 & $0.0002 * * *$ \\
\hline Creatinine, umol/L & $0.32[0.01,1.19]$ & 0.34 & $0.0166^{*}$ \\
\hline Albumin, g/L & $0.84[0.73,0.94]$ & -2.83 & $0.0041 * *$ \\
\hline Protein, g/L & $1.02[0.99,1.11]$ & 1.08 & 0.2563 \\
\hline Alkaline Phosphatase, U/L & $1.03[0.99,1.02]$ & 1.53 & $0.0325 *$ \\
\hline Aspartate Transaminase, U/L & $1.00[1.01,0.99]$ & -0.10 & $0.0011^{* *}$ \\
\hline
\end{tabular}




\begin{tabular}{llcc}
\hline Alanine Transaminase, U/L & $1.00[1.00,0.97]$ & 1.42 & 0.213 \\
Bilirubin, $\mu \mathrm{mol} / \mathrm{L}$ & $0.86[0.75,1.03]$ & -1.62 & 0.4101 \\
Lactate dehydrogenase, U/L & $0.99[0.44,1.58]$ & 11.20 & 0.315 \\
\hline Diabetes mellitus tests & & & 1.52 \\
Glucose, $\mathrm{mmol} / \mathrm{L}$ & $1.06[0.97,1.16]$ & $0.0037 * *$ \\
\hline
\end{tabular}

